\title{
INVESTIGATION OF ROBUST STABILITY FOR DISCRETE-TIME Pol YNOMIals WiTh NONLINEAR UNCERTAinTY STRUCTURE
}

\author{
Radek Matušů
}
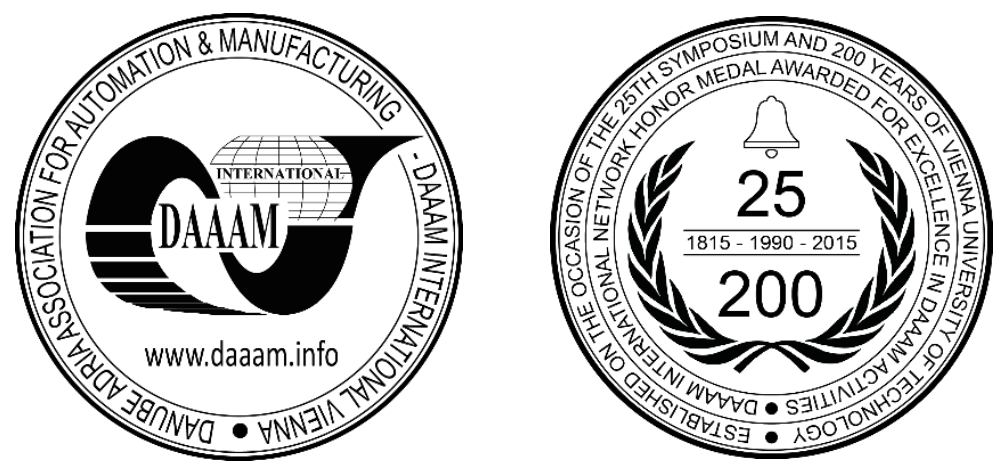

This Publication has to be referred as: Matusu, R[adek] (2016). Investigation of Robust Stability for Discrete-Time Polynomials With Nonlinear Uncertainty Structure, Proceedings of the 27th DAAAM International Symposium, pp.01420146, B. Katalinic (Ed.), Published by DAAAM International, ISBN 978-3-902734-08-2, ISSN 1726-9679, Vienna, Austria

DOI: $10.2507 / 27$ th.daaam.proceedings.021

\begin{abstract}
The main objective of this contribution is to present the application of a graphical approach to robust stability analysis of discrete-time uncertain polynomials with special emphasis to polynomials with a complicated nonlinear uncertainty structure. The generalization of the value set concept and the zero exclusion condition under robust $D$-stability framework is used for this purpose. The relative simplicity of the applied technique is shown by means of the illustrative examples.
\end{abstract}

Keywords: Robust stability; discrete-time polynomial; parametric uncertainty; nonlinear uncertainty structure; value set; zero exclusion condition.

\section{Introduction}

Stability is a critical property of the control loops. In the case of uncertain systems, frequently described by the models with parametric uncertainty, stability must be ensured for all possible members from the assumed family, i.e. so-called robust stability is investigated. Naturally, robust stability represents attractive and deeply studied discipline of the last decades, especially in the continuous-time domain [1], [2], [3], [4], [5], [6]. A number of interesting results have been developed also for the discrete-time systems [7], [8], [9], [10], [11], but many of them are applicable only for simple uncertainty structures (e.g. interval polynomials) and suffer from the lack of generality as they require some restrictions and pre-conditions. And even if some results are more general, they are (in author's opinion), relatively complicated to use.

This contribution is intended to describe the application of the value set concept in combination with the zero exclusion condition under robust $D$-stability framework adopted from [1] for analyzing robust stability of discrete-time polynomials with nonlinear uncertainty structure. The similar ideas as in this work have been already published in the conference paper [12] and its extended version [13]. Moreover, robust stability of continuous-time polynomials with nonlinear uncertainty structure was studied in [14] and robust stability of discrete-time polynomials with a simple uncertainty structure was presented in [15] (the example within the mentioned paper is focused only on discrete-time interval polynomial). 


\section{Problem formulation}

The problem of stability of systems can be supposed as the problem of stability of their characteristic polynomials. The discrete-time polynomial with the uncertain parameters can be written in the form:

$$
p(z, q)=\sum_{i=0}^{n} \rho_{i}(q) z^{i}
$$

where $z$ is the complex variable, $q$ is the vector of uncertainty and $\rho_{i}$ are coefficient functions. Then, the family of polynomials is defined by [1]:

$$
P=\{p(\cdot, q): q \in Q\}
$$

where $Q$ is the uncertainty bounding set (represented by a multidimensional box in this work). Generally, the family of polynomials (2) is robustly stable if and only if $p(\cdot, q)$ is stable for all $q \in Q$, i.e. all roots of $p(z, q)$ must lie within the unit circle. The direct calculation of roots is a possible solution, but it can be very impractical due to potentially extremely long computation times. On that account, the more efficient techniques have to be used.

Among a number of existing methods which were developed and described e.g. in [7], [8], [9], [10], [11], the graphical approach based on combination of the value set concept and the zero exclusion condition under robust $D$-stability framework represents very universal, elegant and powerful tool. Its universality is indispensable especially for the most complicated (nonlinear) uncertainty structures, which are studied in this contribution, because the universal analytical tools practically do not exist for this kind of structures.

\section{Value set concept and zero exclusion condition for robust $D$-stability}

The basic continuous-time version of the value set concept and the zero exclusion condition can be found in [1] and subsequently e.g. in [6]. This contribution employs its improved version, described hereafter, which is extended and generalized to so-called robust $D$-stability framework [1]. The main idea remains the same, but it allows investigating robust stability for an arbitrary stability region $D$, e.g. for a unit circle in case of discrete-time polynomials with parametric uncertainty.

Suppose a family of polynomials (2). The value set at any evaluation point $x \in \mathbf{C}$ is determined by [1]:

$$
p(x, Q)=\{p(x, q): q \in Q\}
$$

In other words, $p(x, Q)$ is the image of $Q$ under $p(x, \cdot)$. For instance, in discrete-time case, substitute $z$ for a point at the unit circle in a family $P=\{p(z, q): q \in Q\}$ and let the vector of uncertain parameters $q$ range over the set $Q$.

In this contribution, the value sets for the families with complicated nonlinear uncertainty structure are plotted by using a suitable sampling (gridding) of the uncertain parameters and direct calculation of related partial points of the value sets for a supposed frequency range. The relative simplicity and applicability for the complex uncertainty structures represents the main advantage of this approach. On the other hand, a long computational time for a high number of uncertain parameters is the weakness.

The zero exclusion condition formulated in [1] says: Let $D$ be an open subset of the complex plane and assume that (2) is a family of polynomials with invariant degree and uncertainty bounding set $Q$ which is pathwise connected. Moreover, suppose that the coefficient functions $\rho_{i}(q)$ are continuous and that (2) has at least one $D$-stable member $p\left(\cdot, q_{0}\right)$. Then (2) is robustly $D$-stable if and only if:

$$
0 \notin p(x, Q)
$$

for all $x \in \partial D$, where $\partial D$ denotes the boundary of $D$.

Moreover, under the assumption that $D$ is bounded (such as unit circle for the case of discrete-time polynomials), the pre-condition of degree invariance is no longer needed.

\section{Illustrative examples}

First, assume the family of discrete-time polynomials with nonlinear (or more specifically polynomial) uncertainty structure: 


$$
\begin{aligned}
& p(s, q)=7 z^{5}+\left(q_{1}^{2}+q_{2}^{2}+5\right) z^{4}+\left(q_{1}^{4}-q_{2}^{2}+5\right) z^{3}+\left(q_{1}^{2}+q_{1} q_{2}^{2}+3\right) z^{2}+\left(2 q_{1}^{3}+3 q_{2}^{3}+2\right) z+\left(3 q_{1}^{2} q_{2}^{2}+1\right) \\
& q_{1}, q_{2} \in[-0.7,0.7]
\end{aligned}
$$

An arbitrary member of the family is taken (e.g. for $q_{1}=q_{2}=0$ ) and its stability is verified. Then, the value sets for 100 points on the unit circle and for sampling both uncertain parameters with the step 0.01 are shown in Fig. 1 . As can be seen, the origin of the complex plane is excluded from the value sets and thus the family (5) is robustly stable.

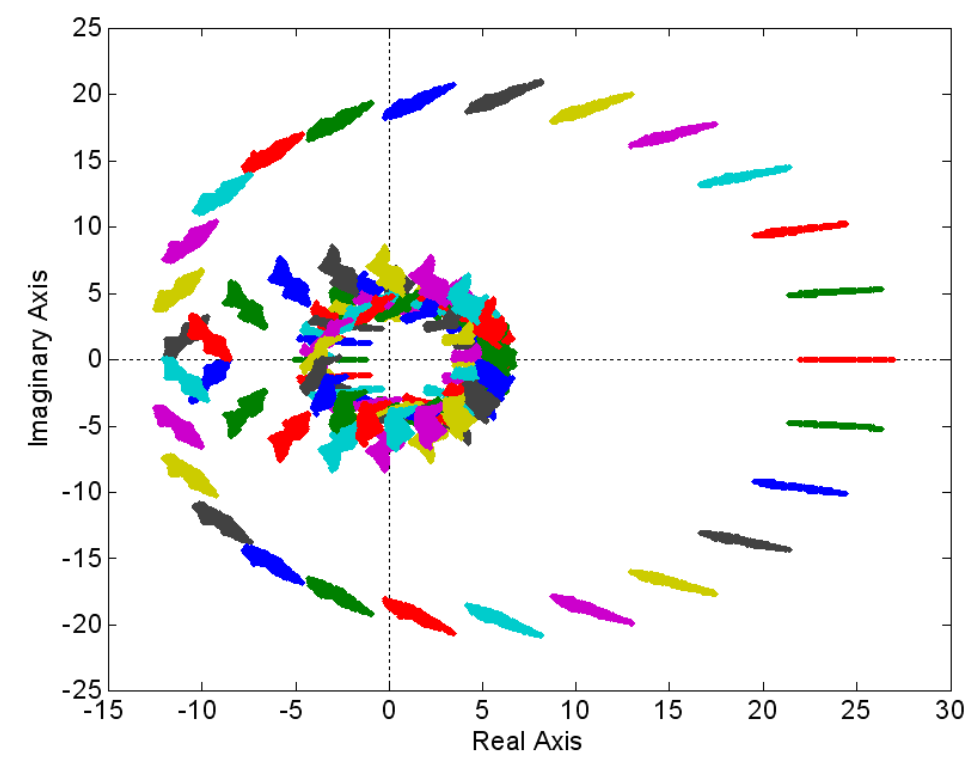

Fig. 1. The value sets of the family (5)

In the next step, the possible intervals for the uncertain parameters in the family (5) are supposed to be wider while the structure itself remains the same, i.e. the following family is considered:

$$
\begin{aligned}
& p(s, q)=7 z^{5}+\left(q_{1}^{2}+q_{2}^{2}+5\right) z^{4}+\left(q_{1}^{4}-q_{2}^{2}+5\right) z^{3}+\left(q_{1}^{2}+q_{1} q_{2}^{2}+3\right) z^{2}+\left(2 q_{1}^{3}+3 q_{2}^{3}+2\right) z+\left(3 q_{1}^{2} q_{2}^{2}+1\right) \\
& q_{1}, q_{2} \in[-1,1]
\end{aligned}
$$

The value sets (for the same sampling as in the previous Fig. 1) are depicted in Fig. 2 (full view) and Fig. 3 (zoomed view for better sight near the origin of the complex plane). The figures clearly prove the robust instability of the family (6) because the complex plane origin is included in the value sets.

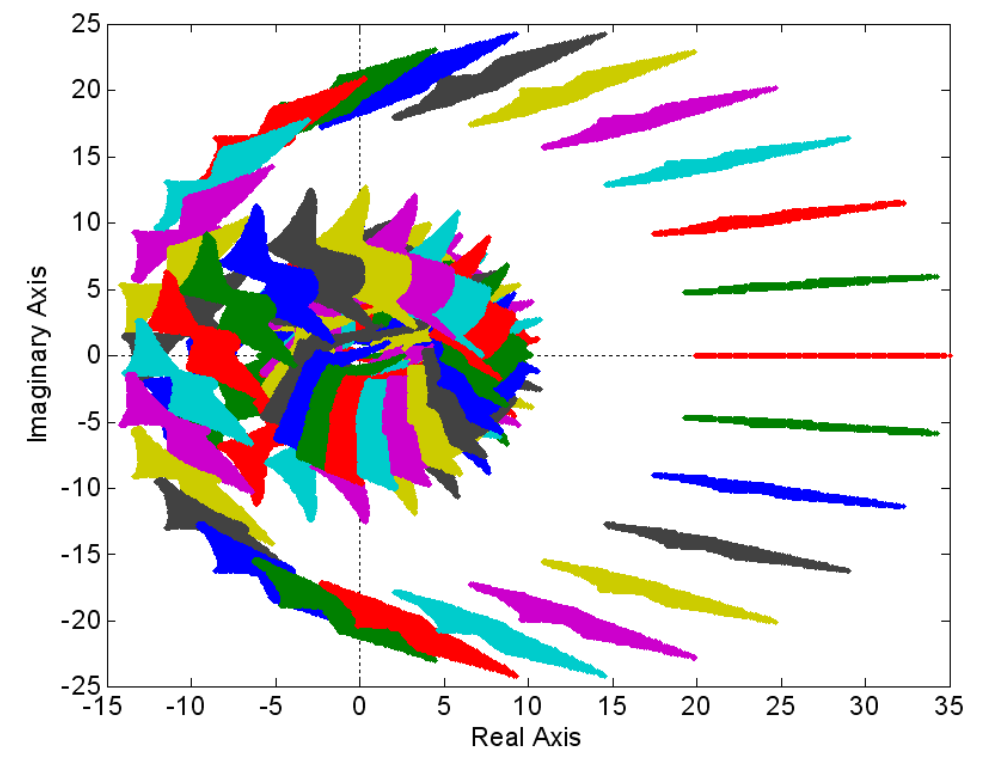

Fig. 2. The value sets of the family (6) - the full view 


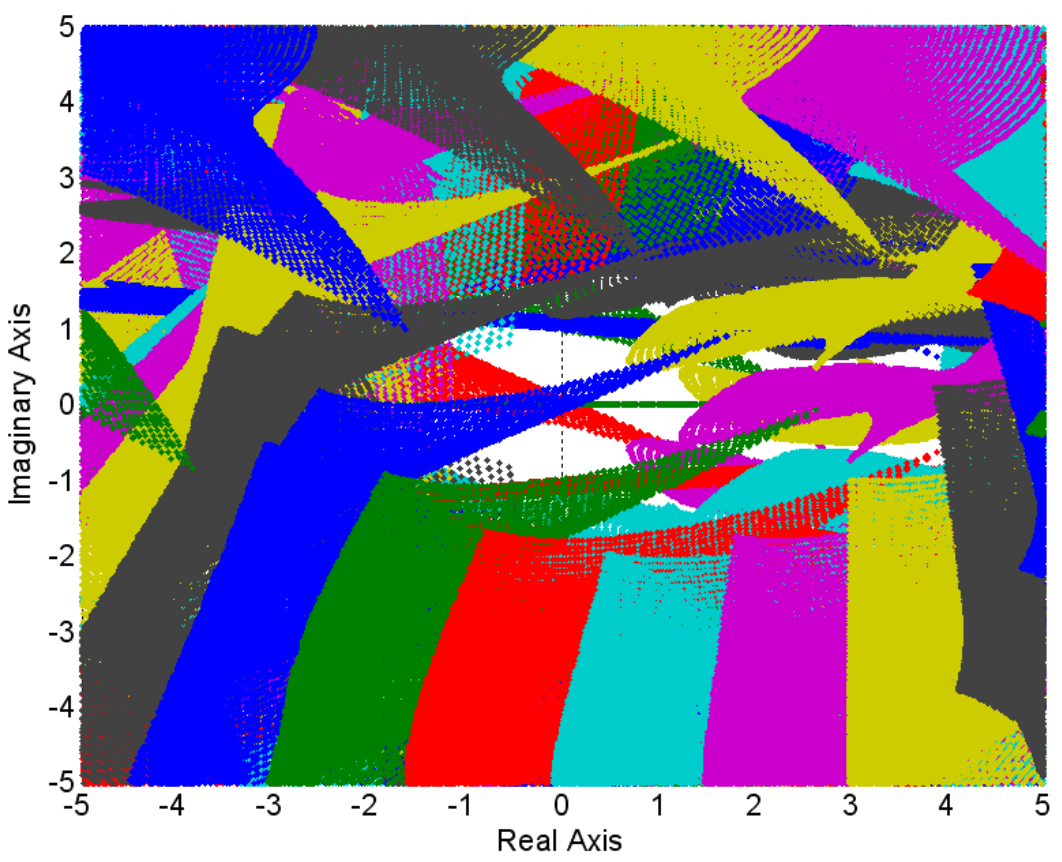

Fig. 3. The value sets of the family (6) - a zoomed view

Finally, assume the family of discrete-time polynomials with nonlinear (now completely general) uncertainty structure:

$$
\begin{aligned}
& p(s, q)=7 z^{3}+\left[\cos \left(q_{1} q_{2}\right)+3\right] z^{2}+\left\lfloor 5 \sqrt{\left|q_{1}\right|}-\sin q_{2}-\cos \left(q_{1} q_{2}\right)+2\right\rfloor z+\left\lfloor-3 \sqrt{\left|q_{2}\right|}+4 \sin q_{1}+2 \cos \left(q_{1} q_{2}\right)+1\right\rfloor \\
& q_{1}, q_{2} \in[-0.3,0.3]
\end{aligned}
$$

As in the previous example, an arbitrary member of the family is taken (e.g. again for $q_{1}=q_{2}=0$ ) and its stability is checked. Subsequently, the value sets for 100 points on the unit circle and for sampling both uncertain parameters with the step 0.005 are shown in Fig. 4. Obviously, the family (7) is stable due to the exclusion of the zero point from the value sets.

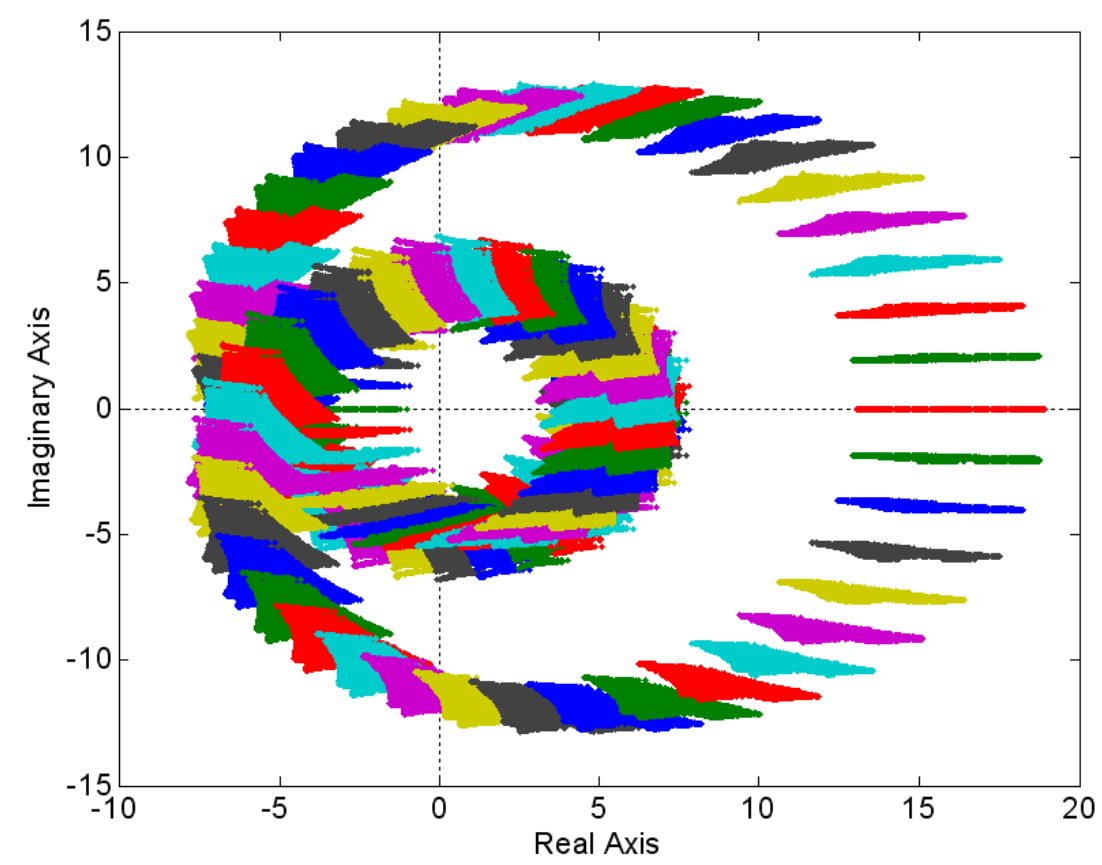

Fig. 4. The value sets of the family (7) 


\section{Conclusion}

This contribution was focused on application of a graphical approach to robust stability analysis of families of discretetime polynomials with special emphasis to families with a complex nonlinear uncertainty structure. The employed technique is based on the generalization of the value set concept and the zero exclusion condition under robust $D$-stability framework. The relative simplicity of the method is demonstrated via the set of the illustrative examples.

\section{Acknowledgments}

This work was supported by the Ministry of Education, Youth and Sports of the Czech Republic within the National Sustainability Programme project No. LO1303 (MSMT-7778/2014).

\section{References}

[1] Barmish, B. R. (1994). New Tools for Robustness of Linear Systems, Macmillan, New York, USA

[2] Bhattacharyya, S. P.; Chapellat, H. \& Keel, L. H. (1995). Robust Control: The Parametric Approach, Prentice Hall, Englewood Cliffs, New Jersey, USA

[3] Ackermann, J.; Bartlett, A.; Kaesbauer, D.; Sienel, W. \& Steinhauser, R. (1993). Robust Control - Systems with Uncertain Physical Parameters, Springer-Verlag, London, Great Britain

[4] Dullerud, G. E. \& Paganini, F. (2000). A Course in Robust Control Theory. A Convex Approach, Springer, New York, USA

[5] Sánchez-Peña, R. S. \& Sznaier, M. (1998). Robust Systems: Theory and Applications, John Wiley \& Sons, New York, USA

[6] Matušů, R. \& Prokop, R. (2011). Graphical analysis of robust stability for systems with parametric uncertainty: an overview. Transactions of the Institute of Measurement and Control, Vol. 33, No. 2, pp. 274-290

[7] Kraus, F. J.; Anderson, B. D. O. \& Mansour, M. (1988). Robust Schur polynomial stability and Kharitonov's theorem. International Journal of Control, Vol. 47, No. 5, pp. 1213-1225

[8] Mansour, M.; Kraus, F. J. \& Anderson, B. D. O. (1988). Strong Kharitonov theorem for discrete systems, Proceedings of the 27th Conference on Decision and Control, Austin, Texas, USA

[9] Barmish, B. R. (1989). An Extreme Point Result for Robust Stability of Discrete-Time Interval Polynomials, Proceedings of the 28th Conference on Decision and Control, Tampa, Florida, USA

[10] Kraus, F., Mansour, M. \& Jury, E. I. (1992). Robust Schur Stability of Interval Polynomials. IEEE Transactions on Automatic Control, Vol. 37, No. 1, pp. 141-143

[11] Jetto, L. \& Orsini, V. (2008). Some remarks on the Schur stability of interval polynomials, Proceedings of the 16th Mediterranean Conference on Control and Automation, Ajaccio, France

[12] Matušů, R. \& Prokop, R. (2013). Graphical Approach to Robust Stability Analysis for Discrete-Time Interval Polynomials, Proceeding of the 15th WSEAS International Conference on Mathematical and Computational Methods in Science and Engineering, Kuala Lumpur, Malaysia, pp. 34-37

[13] Matušů, R. (2014). Robust Stability Analysis of Discrete-Time Systems with Parametric Uncertainty: A Graphical Approach. International Journal of Mathematical Models and Methods in Applied Sciences, Vol. 8, pp. 95-102

[14] Matušů, R. \& Prokop, R. (2009). Systems with Nonlinear Uncertainty Structure: Robust Stability Analysis, Annals of DAAAM for 2009 \& Proceedings of 20th International DAAAM Symposium, Vienna, Austria, pp. 1373-1374

[15] Matušů, R. (2010). A Graphical Approach to Robust Stability Analysis of Discrete-Time Systems with Parametric Uncertainty, Annals of DAAAM for 2010 \& Proceedings of 21st International DAAAM Symposium, Zadar, Croatia, pp. $485-486$ 